\title{
TERMOGRAFIA POR INFRAVERMELHO EM MEDICINA VETERINÁRIA
}

\author{
Kamilla Dias Ferreira ${ }^{1}$, Saulo Humberto Ávila Filho ${ }^{2}$, Jéssica Fernanda \\ Bertolino ${ }^{3}$,Luiz Antônio Franco da Silva ${ }^{4}$,Valcinir Aloisio Scalla Vulcani ${ }^{5}$ \\ ${ }^{1}$ Mestranda, Escola de Veterinária e Zootecnia da Universidade Federal de Goiás, \\ Goiânia, Brasil. e-mail: kamilladiasvet@gmail.com \\ ${ }^{2}$ Mestrando,Escola de Veterinária e Zootecnia - UFG. e-mail: \\ ${ }^{3}$ Mestranda,Escola de Veterinária e Zootecnia - UFG \\ ${ }^{4}$ Professor Doutor- Escola de Veterinária e Zootecnia - UFG - Goiânia, GO \\ ${ }^{5}$ Professor Doutor -Curso de Medicina Veterinária, Universidade Federal de Goiás, \\ Campus de Jataí, Jataí (GO).
}

Recebido em: 08/04/2016 - Aprovado em: 30/05/2016 - Publicado em: 20/06/2016 DOI: 10.18677/Enciclopedia_Biosfera_2016_115

\begin{abstract}
RESUMO
O exame termográfico consiste na mensuração da energia infravermelha empregando câmeras que captam as radiações emitidas pelo corpo de um animal. As imagens capturadas através do termógrafo geram os termogramas, que são imagens que se diferem de acordo com a distribuição de temperatura. A diferença de temperaturas existentes entre dois corpos é visível pelas imagens exibidas pelo aparelho termográfico. Cores em tons de azul e roxo indicam que a temperatura é baixa, enquanto que as cores em tons mais quente, variando do vermelho ao amarelo, indicam uma temperatura mais elevada. Fatores devem ser levados em consideração, como a preparação do paciente e do ambiente onde se realizará o exame, evitando mensurações inadequadas. $O$ uso da termografia expandiu-se para diversas especialidades na medicina veterinária, como método auxiliar para diagnósticos de processos inflamatórios em diferentes espécies de animais, assim como na detecção precoce de enfermidades ao ser utilizado em animais de difícil manejo.
\end{abstract}

PALAVRAS- CHAVE: Infravermelho, Medicina Veterinária. Termografia

\section{THERMOGRAPHY IN INFRARED IN VETERINARY MEDICINE}

\begin{abstract}
The thermographic examination consists of measuring the infrared energy using cameras that capture the radiation emitted by the body of an animal. The images captured by the thermograph generate thermograms that are images that differ according to the temperature distribution. The difference in temperature existing between two bodies is visible the images captured by thermographic machine. Colors shades of blue and purple indicates that the temperature is low, while the hot color shades between red and yellow, indicating a higher temperature. Factors must be taken into account, such as the preparation of the patient and the environment where it will conduct the examination, avoiding inadequate measurements. The use of ENCICLOPÉDIA BIOSFERA, Centro Científico Conhecer - Goiânia, v.13 n.23; p.1298 2016
\end{abstract}


thermography has expanded to various specialties in veterinary medicine, as a method for inflammatory processes diagnosis in different species of animals, as the early detection of diseases when used in unwieldy animals.

KEYWORDS: Thermography, Infrared, Veterinary Medicine.

\section{INTRODUÇÃO}

$\mathrm{Na}$ medicina veterinária, os meios de diagnósticos têm apresentado avanços substanciais, empregando técnicas cada vez mais elaboradas e seguras que permitem ao médico veterinário realizar uma abordagem clínica cada vez mais completa e eficiente (LAHIRI et al., 2012). Dentre os métodos mais inovadores se insere a termografia, uma técnica de mensuração da temperatura da superfície do corpo, em diferentes níveis, como uma forma de contribuir para a avaliação clínica do paciente (MIKAIL, 2010).

Uma das vantagens da termografia é o auxílio na percepção e localização de focos específicos de anormalidades teciduais, evidenciando o grau de comprometimento dos mesmos. Na reação inflamatória o calor é um dos sinais que indicam a presença desse processo, sendo que, antes mesmo que os sinais clínicos apareçam no paciente é possível, por intermédio desse método de diagnóstico, observar os indícios do processo inflamatório (VIANNA \& CARRIVE, 2005).

É importante salientar que existem fatores que podem influenciar o resultado de exames, de um modo geral. No que tange a termografia, alguns pontos negativos são os falsos negativos quando o exame é realizado sob exposição à radiação solar e sujidades. Outros fatores como o umedecimento e espessura da pele e a densidade da pelagem também devem ser considerados (RING, 2000; ROBERTO \& SOUZA, 2014). Porém, o exame se enquadra nos métodos que promovem o bem-estar animal, tendo em vista a necessidade de adequação de técnicas e do uso de equipamentos que tornem os procedimentos na medicina veterinária menos invasivos, proporcione maior conforto ao paciente e reduza o estresse provocado ao animal (ROBERTO \& SOUZA, 2014).

Considerando as diversas possibilidades de uso da termografia como método auxiliar de diagnóstico na medicina veterinária, acredita-se que o assunto necessite ser amplamente discutido. Assim, pela sua eficácia e fácil aplicabilidade em diferentes espécies de animais pode ser uma importante alternativa empregada na obtenção de dados com exatidão e precisão. Esta revisão objetivou reunir informações a respeito da termografia de radiação infravermelha, com relação a sua importância histórica até os dias atuais em várias áreas de atuação. No que se diz a respeito do uso desse método de diagnóstico complementar, justifica-se principalmente pela eficácia e fácil aplicabilidade em diferentes espécies de animais sendo uma importante alternativa por realizar a obtenção de dados com exatidão e precisão.

\section{Histórico da termografia}

Relatos relacionados a termografia apontam que o método surgiu a partir de estudos feitos pelo médico e filósofo grego Hipócrates (RING, 2000; LAHIRI et al., 2012). A mensuração da temperatura era realizada utilizando o dorso da mão para sentir o calor em diferentes partes do corpo. Utilizava-se também a lama, que nos locais que secassem mais rapidamente, após a aplicação, seria o de maior temperatura. Seguindo essa linha de raciocínio Hipócrates emitia diagnósticos, se havia ou não a presença de alguma enfermidade, baseando-se nas variações da temperatura corporal do paciente (LAHIRI et al., 2012). 
Em 1800, William Herschel, realizou a descoberta da radiação térmica infravermelha utilizando um espectroscópio. Descobriu-se que o sol emitia raios infravermelhos e assim realizou estudos das temperaturas das diferentes faixas dos espectros que compõem a luz visível. Entretanto, esta descoberta e sua relação com a luz não se tornaram claras até a metade do século XIX (BRIOSCHI et al., 2003). Em 1840 o filho de Willian Herschel, John Herschel um dos pioneiros no campo da fotografia, realizou as primeiras imagens utilizando o sistema infravermelho por meio da técnica evaporográfica. Esta técnica consistia na evaporação do álcool obtido de uma superfície pintada com carbono. Diferentes abordagens, com relação a essa descoberta, ainda foram realizadas em meados de 1850, por inúmeros estudiosos como Becquerel, Golay e Czerny. Cada técnica produziu termogramas com discriminação insuficiente de temperatura para o uso que se destinavam (RING, 2000).

Novos avanços da tecnologia de radiação infravermelha foram relatados no período da Segunda Guerra Mundial, em que o seu uso era restrito para fins militares, empregados no sistema de visão noturna para detectar alvos inimigos (BRIOSCHI et al., 2003). Em medicina veterinária, a medicina equestre foi uma das áreas pioneiras a utilizar esse tipo de exame complementar nos diagnósticos de diversas lesões e afecções. Entretanto, o exame termográfico vem crescendo ao longo dos anos não limitando seu uso apenas a espécie equina, mas também abrangendo o uso em cães, gatos e em animais de produção (DE LIMA et al., 2013).

\section{O aparelho termográfico}

Os termógrafos são instrumentos de captação de radiação infravermelha que detectam pequenas oscilações térmicas, do objeto ou corpo analisado, por apresentarem grande sensibilidade e alta resolução (FIGUEIREDO et al., 2012). Após a captação da radiação infravermelha, são gerados os termogramas que são mapas térmicos de imagens com diferentes distribuições de temperatura (RING, 2000).

Os termogramas permitem a distinção da distribuição do calor emitido pelo elemento analisado, estabelecendo medidas precisas de temperatura e a identificação das curvas isotérmicas (FIGUEIREDO et al., 2012). Estes valores são visualizados em uma imagem que pode ser multicolorida ou monocromática, em tons de cinza, dependendo da escala de cores selecionada. As imagens digitalizadas são facilmente visualizadas e podem ser analisadas usando programas de computadores específicos, responsáveis pelo mapeamento térmico. As cores das imagens emitidas pelo termograma indicam se a temperatura de um determinado local está dentro dos padrões de normalidade esperados (LAHIRI et al., 2012).

Qualquer corpo ou objeto que apresente temperatura acima de zero absoluto emite radiação infravermelha devido à movimentação interna das moléculas (MIKAIL, 2010). Todavia, essa emissividade, no caso dos seres vivos, sofre a influência de diversos fatores como a presença de ar, umidade e circulação sanguínea. Considera-se que à medida que a circulação sanguínea periférica aumenta, a emissividade da radiação também aumenta, sendo perceptível no termógrafo. $\mathrm{O}$ aparelho demonstra a região em que ocorre a alteração como sendo a que apresenta uma temperatura mais alta em relação às circunvizinhas, através do espectro de cores aplicado às imagens (DE LIMA et al., 2013). 


\section{A análise quantitativa e a interpretação de imagens}

A termografia quantitativa é uma proposta de análise dos dados do termograma que fornece informações objetivas no acompanhamento de afecções e tratamentos ao longo do tempo (RING, 1990). De um modo geral, em casos que se realiza um único exame, o indicado é que o profissional realize o exame comparando a superfície com suspeita de lesão com a mesma região contralateral (BRIOSCHI et al., 2003). Nas avaliações seriadas no tempo, ou seja, a análise quantitativa deve-se realizar a padronização das temperaturas obtidas nos dias subsequentes em relação à primeira avaliação, de forma a poder compará-las e acompanhar a evolução do paciente (BASILE et al., 2010; CHRISTENSEN et al., 2012).

Com relação ao controle da temperatura ambiente, o período de aclimatação dos animais antes da realização do exame termográfico deve ser considerado. Em especial na espécie equina, em que o preconizado pela literatura é um período de aclimatação do local variando entre 39 e 60 minutos, antes da realização das termografias (SANCHEZ et al., 2008; SIMON et al., 2006). Considerase que na prática estas limitações de procedimento, para obtenção de avaliações seriadas no tempo, dificultam a aplicação da termografia quantitativa na medicina veterinária, em especial na medicina de equinos (TUNLEY \& HENSON, 2004). Para realização do exame, torna-se muito difícil obter uma sala ou baia com controle de temperatura constante para manter os cavalos por períodos de no mínimo 60 minutos (BASILE et al., 2010).

O mapeamento térmico, ou seja, a interpretação das imagens é realizada através de software que permite a mensuração e análise da temperatura absoluta, máxima, média e mínima da região de interesse. Deste modo é possível definir essa região por intermédio do uso de spots ou de áreas como meio para analisar locais específicos (MALDAGUE , 2002).

\section{Preparação do paciente}

A preparação do paciente é de extrema importância para que não ocorra a mensuração inadequada durante a realização do exame termográfico. Para tanto é necessário evitar que a região avaliada esteja úmida, e para minimizar a chance dessa situação ocorrer não submeter o animal a banhos e duchas quentes antes do procedimento (BAILEY et al., 2004; VICARI NOGUEIRA, 2014). A transmissão das ondas de infravermelho sofre interferência quando há presença de gotículas de água superficiais que atuam como uma barreira impedindo que partes dessas ondas cheguem até o receptor do equipamento. Além disso, auxiliam na perda de calor superficial por evaporação, interferindo na temperatura local da superfície (LOUGHIN \& MARINO, 2007).

O uso de agentes tópicos, como os géis à base de cânfora e pomadas interferem diretamente na temperatura local devendo ser evitados pelo menos 12 horas antes do exame (EDDY et al., 2001). O paciente deve estar em jejum até três horas antes do procedimento e deve permanecer por no mínimo 15 minutos no ambiente para que a pele entre em equilíbrio térmico com a temperatura da sala. A prática de exercícios ou fisioterapia também deve ser evitada até duas horas antes do exame (LOUGHIN \& MARINO, 2007). 


\section{Influencia da distância e do ambiente e na aquisição de imagens}

Existe uma distância considerada ideal para realização do exame termográfico. $O$ indicado é verificar a distância na primeira avaliação para repetir sempre a mesma distância nos exames seguintes (MALDAGUE, 2002). Ao avaliar este parâmetro deve-se considerar a área que abrange toda a superfície a ser examinada, incluindo uma porção marginal da região não lesionada (RING, 1990). Ao realizar o procedimento deve-se atentar ao posicionando do equipamento, de modo que não fique muito distante do corpo ou objeto que se deseja obter a imagem, pois ocorrerá a perda da resolução da mesma, visto que as temperaturas são fornecidas para cada pixel da imagem (DE LIMA et al., 2013; MALDAGUE, 2002). É válido ressaltar que variações na distância de aquisição de imagens podem comprometer as avaliações comparativas subsequentes realizadas no acompanhamento da evolução do paciente (TUNLEY et al., 2004).

Com relação ao ambiente para realização do exame, deve estar padronizado a uma temperatura constante, sendo indicado pela literatura entre $20^{\circ} \mathrm{C}$ a $2^{\circ} \mathrm{C}$, não sendo superior a $30^{\circ} \mathrm{C}$ (MIKAIL, 2010). É importante também evitar trocas térmicas abruptas, o uso de lâmpadas fluorescentes e impedir a entrada de luz solar no local de realização do exame. O ambiente controlado é de extrema relevância para a realização do exame termográfico, sendo necessário a padronização das temperaturas em relação às variações térmicas do ambiente, visando eliminar os efeitos ambientais sobre a superfície corpórea (MALDAGUE, 2002) .

\section{TERMOGRAFIA COMO MÉTODO DE AUXÍLIO NOS DIAGNÓSTICOS}

A termografia é uma técnica de registro gráfico não invasivo, em que são detectados padrões térmicos gerados pela emissão infravermelha de calor da superfície corporal (INFERNUSO et al., 2010). Os locais na pele em que estão presentes anormalidades em áreas de vascularização denominam-se pontos quentes, hot spots, que podem ser observados em casos de inflamação aguda. Em contrapartida, os pontos frios, cold spots, podem ser observados nas regiões de necrose (BAILEY et al., 2004).

Devido ao calor ser um dos sinais cardiais da inflamação, com a utilização do exame termográfico é possível observar os indícios do processo inflamatório antes mesmo que os sinais clínicos apareçam no paciente (SIMON et al., 2006). O calor da pele é baseado na circulação local e, portanto, variações na temperatura podem indicar processos de ruptura da homeostase (VIANNA \& CARRIVE, 2005). Trata-se de uma ferramenta capaz de evidenciar afecções com quaisquer tipos de alteração de perfusão sanguínea, tais como inflamações, tumores, fibroses, neuropatias ou isquemias. Permite o diagnóstico de problemas relacionados a tendões, ligamentos, articulações, músculos e ossos, desde que os últimos sejam cobertos por fina musculatura (BRIOSCHI et al., 2003).

É importante ressaltar que este procedimento não é indicado para a realização de um diagnóstico definitivo, e sim como um método de exame complementar utilizado para auxiliar o diagnóstico clínico (LAHIRI et al., 2012). O seu uso ainda é considerado uma tecnologia emergente, mas que apresenta alguns benefícios com a utilização, como a rapidez com que a técnica pode ser executada e pelo fato do procedimento ser indolor, não sendo necessário o uso de sedativos ou 
contraste, e por não expor o paciente a radiações ionizantes (BRIOSCHI et al., 2003; LAHIR et al., 2012).

\section{Evolução da termografia na medicina veterinária}

O exame termográfico é empregado desde 1970 tanto em humanos quanto em animais, sendo que inicialmente só era utilizado na detecção de lesões inflamatórias em equinos de corrida (CETINKAYA \& DEMIRUTKU, 2012). Entretanto, somente nos últimos anos, com os avanços alcançados no aprimoramento do aparelho, e consequentemente redução dos custos dos equipamentos, o seu uso encontra-se em expansão na medicina veterinária (ROBERTO \& SOUZA, 2014). O emprego do aparelho termográfico é um complemento importante das outras modalidades de diagnóstico por imagem, como a tomografia computadorizada. Entretanto, em alguns casos, a termografia demonstra-se mais eficiente ao detectar precocemente doenças ainda em estágios iniciais, o que muitas vezes não é observado em outras técnicas de imagem (TURNER et al., 2001; INFERNUSO et al., 2010).

Entre os grandes desafios, relacionados ao uso da termografia como meio de diagnóstico, encontra-se a falta de especificidade em identificar o local afetado com precisão (ARORA et al., 2008). O que se observa nas imagens termográficas, de um modo geral, são alterações no padrão térmico indicando a anormalidade presente, que retorna aos padrões adequados de normalidade ao ser instaurado o tratamento adequado (RING, 1990).

\section{APLICAÇÕES DA TERMOGRAFIA NA MEDICINA VETERINÁRIA}

A termografia vem sendo utilizada como ferramenta complementar no diagnóstico de diversas enfermidades (FIGUEIREDO et al., 2012). Na medicina, o início procedeu na área da oncologia, mediante observações em tumores de mama, visto que ocasionavam a elevação da temperatura da pele. No que tange a medicina veterinária, há mais de três décadas a termografia é aplicada para detectar precocemente lesões músculo-esqueléticas em equinos (SIMON et al., 2006). O exame termográfico foi difundido, principalmente, pelos estudos realizados nesta espécie, na área de ortopedia, sendo que essa prática vem crescendo, principalmente, ao longo dos últimos anos (TURNER \& EDDY, 2001).

O uso da termografia é observado tanto na área clínica assim como nas pesquisas. Por se tratar de um exame de imagem, durante a execução da técnica deve ser considerado a sensibilidade do aparelho, assim como a presença de artefatos que podem prejudicar a análise dos dados (VIANNA \& CARRIVE, 2005). Compete ao profissional conhecimento ao manusear o aparelho de forma correta para obtenção dos resultados esperados (TURNER \& EDDY, 2001; VIANNA \& CARRIVE, 2005). Ao executar o procedimento de forma correta a termografia se apresenta como um exame auxiliar relevante, auxiliando o profissional em um diagnóstico mais preciso de doenças, ainda no inicio do curso (MALDAGUE, 2002).

\section{ANIMAIS DE PRODUÇÃO}

\section{Aves e Suínos}

A radiação infravermelha detectada pelo aparelho termográfico pode ser de grande valia para observar o nível de estresse em animais de produção, já que esta ferramenta pode detectar alterações no fluxo sanguíneo (STEWART et al., 
2005). A câmera termográfica possui precisão, pois detecta variações mínimas de temperatura, que vão do quente ao frio, que por sua vez, indicam anormalidades fisiológicas em determinado animal (TURNER \& EDDY, 2001; INFERNUSO et al., 2010). Devido à pele ser o principal órgão de termorregulação dos animais homeotérmicos, considera-se que esse processo é um fator importante a ser observado durante a avaliação termográfica. Deste modo deve-se analisar a condição de conforto térmico dos animais, pois quando encontram-se em hipotermia ou hipertermia podem diminuir a eficiência do exame (EDGAR et al., 2013).

Em estudo realizado, foi avaliado o estresse induzido por hipertermia em galinhas. Observou-se que em um período de 10 a 15 minutos do inicio do momento de estresse houve um aumento entre 0,5 a $1,5^{\circ} \mathrm{C}$ na temperatura corpórea desses animais. Levando em consideração a elevação da temperatura provocada pelo momento de estresse, considerou-se a termografia como um método em potencial para avaliar o estresse induzido em galinhas (FIGUEIREDO et al., 2008; EDGAR et al., 2013). Em outro estudo foi analisada a eficiência do aparelho termográfico na detecção da variação de produção de calor metabólico de pintos na fase de crescimento. Esses animais foram alimentados com diferentes densidades energéticas, de modo que foi verificado que a termografia por infravermelho identificou efetivamente a atividade metabólica dessas aves (FERREIRA et al., 2011).

Em suínos por meio de um sistema com uso da termografia, observou-se que nos últimos dias de gestação das fêmeas e particularmente após o parto, a temperatura da pele sobre a glândula mamária representava a área mais quente da superfície corporal. No primeiro dia de lactação, a temperatura superficial da glândula mamária foi de $3^{\circ} \mathrm{C}$ e nos períodos posteri ores da lactação, a temperatura localizou-se entre 37 e $38^{\circ} \mathrm{C}$. As imagens termográfi cas também foram eficazes para avaliar o conforto térmico dos animais nas instalações, demonstrando que as temperaturas superficiais são significativamente afetadas pela temperatura ambiente (KOTRBACEK \& NAU, 1985). Ainda na espécie suína, uma das afecções mais prevalentes nos sistemas intensivos de produção são as artrites. Essa enfermidade afeta consideravelmente o desempenho desses animais e o bem-estar. Em um estudo de caso, buscou-se avaliar a eficiência do uso das imagens termográficas para identificação de artrite, ou de lesões em suínos por meio da interpretação dos termogramas de temperaturas superficiais obtidas. Foram utilizados cinco animais com suspeita de artrite nos membros posteriores. Os valores médios da temperatura superficial foram menores no membro sadio, com $33,6^{\circ} \mathrm{C}$, do que nos que apresentavam a enfermidade, com $35,6^{\circ} \mathrm{C}$ evidenciando que por meio de imagens termográficas, é possível a identificação de processos inflamatórios em suínos (GRACIANO et al., 2014).

\section{Ruminantes}

Em estudo experimental realizado em ovelhas, que foram infectadas com o vírus da febre catarral maligna, a termografia mostrou-se como um método de diagnóstico complementar eficiente para a avaliação desta enfermidade nos animais infectados com o vírus (PEREZ DE DIEGO et al., 2013). Em bovinos, a detecção do estado infeccioso e febril em animais acometidos pelo complexo respiratório bovino foi o objeto de outro estudo realizado. Foi utilizado o aparelho termográfico próximo aos bebedouros dos animais para verificação dos valores de temperatura. Observaram variações nos valores da temperatura significativamente maior em 
animais acometidos com a enfermidade, ressaltando a importância da técnica como um exame de triagem (SCHAEFER et al., 2007). Ainda na espécie bovina, foram analisados através de imagens captadas pelo aparelho de termografia por infravermelho, cascos de vacas infectadas com o vírus da febre aftosa. Os termogramas indicaram um aumento acentuado na temperatura do casco dos animais acometidos por esta doença, antes de apresentarem os sintomas clínicos. Outros estudos utilizando os termógrafos demonstraram a existência de mudanças constantes na temperatura do casco em caprinos. Os autores concluíram que a utilização da termografia infravermelha pode efetivamente detectar os distúrbios na circulação sanguínea periférica (RAINWATER-LOVETT et al., 2009).

Também foram realizadas pesquisas envolvendo estresse em animais de produção e características qualitativas da carne avaliando o estresse ante-mortem. Os autores deste estudo verificaram com o uso do aparelho termográfico, bovinos transportados por períodos de 1,5 horas. Imagens térmicas da região dorsal desses animais foram captadas com temperaturas de 36,2 e $37,2{ }^{\circ} \mathrm{C}$, no momento antes do transporte e após o transporte, respectivamente.Verificou-se, deste modo, a existência de algum grau de carne PSE (pálida, macia e exsudativa) ou DFD (escura, firme e seca) no momento do abate, como indicativo de estresse sofrido pelos animais (SCHAEFER et al., 2001).

\section{Equinos}

Estudos demonstraram que a câmera de infravermelho apresentou eficácia na detecção de lesões que acometem equinos (TURNER \& EDDY, 2001). Com relação ao aparecimento da tendinite em cavalos de corrida, os resultados indicaram pontos quentes antes mesmo que houvesse evidência clínica de edema e claudicação, demonstrando que a termografia auxilia no diagnóstico precoce, portanto, assegurando o estado de saúde do animal antes de ser exposto a atividades físicas intensas como a corrida (FIGUEIREDO et al., 2012).

Outra vantagem com a utilização do aparelho termográfico é avaliar o equilíbrio do casco de equinos. Observa-se que a medida que o casco está desnivelado, atingindo o chão de forma desuniforme, gera-se um calor desigual devido ao atrito do casco desnivelado com o solo (INFERNUSO et al., 2010). Deste modo, através do exame termográfico em equinos consegue-se obter informação rápida e precisa sobre a laminite, abscessos subsolares ou submurais, hematomas e outras condições inflamatórias do casco, especialmente quando os resultados da clínica e da radiografia são inconclusivos (TURNER et al., 2001; INFERNUSO et al., 2010). De um modo geral, mudanças difusas no padrão térmico da parede do casco são relevantes no diagnóstico da laminite (ROSS \& DYSON, 2003). Observa-se uma tendência da banda coronária ser a zona mais quente, fator que interfere em um diagnóstico mais preciso nesta área. O esperado é que a região da banda coronária esteja representada pela cor vermelha e os cascos pelo amarelo ou verde (EDDY et al., 2001). Em geral, a parede do casco passa a ter temperaturas próximas as da banda coronária, apontando alguma anormalidade como um processo inflamatório (EDDY et al., 2001).

O indicado é que todos os cascos avaliados nos animais sejam comparados entre si, sendo que uma diferença maior que $1^{\circ} \mathrm{C}$ entre eles pode ser sugestivo de anormalidade (ROSS \& DYSON, 2003; INFERNUSO et al., 2010). Outra afecção importante na clínica médica de equinos é a síndrome do navicular. Os problemas detectados no osso navicular se apresentam com o aumento da 
temperatura nos cascos, que indica a lesão, é evidenciado tanto na vista palmar e plantar, entre os bulbos do talão (EDDY et al , 2001).

A região dorsal do cavalo também é possível de ser avaliada através do conhecimento do padrão térmico normal. É possível identificar as alterações presentes por meio de um mapa térmico. Este mapa é um traçado evidenciado pelo exame termográfico que percorre desde a região da nuca até à base da cauda. Essa região é conhecida como "linha central", e se apresenta com temperaturas mais elevadas (HENSON, 2009; INFERNUSO et al., 2010). Observa-se a formação de linhas isotérmicas de temperatura decrescentes à medida que se afasta da região da coluna. Na sequência, outro padrão térmico é traçado na região da garupa. Esse traçado consiste em uma linha isotérmica quente conhecida com "padrão em "T" que junta as duas tuberosidades coxais entre elas e a linha central (HENSON, 2009).

$\mathrm{Na}$ reprodução de equinos, o uso do aparelho termográfico demonstrou eficiência na distinção de éguas prenhas das não prenhas, em animais com cinco semanas antes do parto. Segundo os autores deste estudo, os animais com útero gravídico apresentaram maiores temperaturas na região do abdômen, quando comparados a animais com úteros não gravídicos, independente das condições ambientais. Entretanto, a diferença ficou mais aparente quando a temperatura ambiente foi inferior a $19{ }^{\circ} \mathrm{C}$ (BOWERS et al., 2009).

\section{ANIMAIS DE COMPANHIA}

\section{Cardiologia Veterinária}

O exame termográfico foi utilizado para determinar a localização anatômica de um trombo arterial em um cão de oito anos de idade que apresentou paralisia aguda do membro pélvico direito. O termograma indicou considerável redução de temperatura na região abaixo da origem da artéria femoral profunda direita em relação ao membro pélvico esquerdo. A confirmação foi realizada por incisão local da artéria seguida de tratamento, concluiu-se que a utilização da termografia como exame, pode auxiliar no diagnóstico e monitoramento em casos de trombose arterial canina (KIM \& PARK, 2012).

\section{Odontologia Veterinária}

Em estudo inédito realizado na área de odontologia veterinária com aplicação da termografia, buscou-se diagnosticar a doença periodontal e o abscesso periapical com essa ferramenta de suporte. O exame termográfico foi utilizado na detecção da doença periodontal em diferentes graus, através da temperatura da gengiva. Observou-se que este meio de diagnóstico não se mostrou eficiente em comparação aos exames rotineiros, pois não houve diferença significativa da temperatura gengival nos diferentes graus da doença (LANGE et al., 2014).

O mesmo estudo ainda avaliou a termografia como método diagnóstico na verificação da presença ou não de abscesso periapical através da temperatura gengival, utilizando como comparativo a radiografia intra-oral. Nesta enfermidade analisada foi observado um aumento significativo da temperatura da gengiva na presença de abscesso periodontal. Concluiu-se que a termografia demonstrou ser uma ferramenta eficiente na detecção dos abscessos periapicais em cães, quando mensurada a temperatura da gengiva adjacente à raiz dentária acometida, podendo 
ser utilizada como uma ferramenta de triagem para a confirmação diagnóstica pela radiologia (LANGE et al., 2014).

\section{Oncologia Veterinária}

$\mathrm{Na}$ medicina veterinária percebe-se 0 constante crescimento nas ocorrências de afecções oncológicas em animais domésticos. Em cadelas, o tumor de mama é o tipo mais diagnosticado, sendo justificado o incremento do número de casos devido a maior expectativa de vida observada nos animais (REIS et al., 2010). Como acontece no tumor mamário humano, no animal, pode-se detectá-lo precocemente com o uso do aparelho termográfico, visto que fica evidenciada a ocorrência da enfermidade pelas variações de temperatura. Estas variações estão relacionadas a modificações do fluxo sanguíneo e do aumento do metabolismo das células mamárias (MALDAGUE, 2002; ARORA et al., 2008). As células cancerígenas animais também tendem a produzir óxido nítrico, sendo este a fonte estimuladora da angiogênese e da vasodilatação que se encontram presentes nas neoplasias (ARORA et al., 2008). O uso da termografia desempenha papel de localizar as áreas de inflamação agudas ou crônicas e, também pode ser utilizada com relação ao diagnóstico precoce de tumores de mama, mesmo que ainda não observados clinicamente (BASILE et al , 2010).

Em estudo realizado por REIS et al. (2010), quatro cadelas foram selecionadas objetivando avaliar os indícios da correlação entre as imagens ultrassonográficas e termográficas, com a classificação dos tumores de mama das cadelas. Não foi observada relação direta entre o padrão de imagem e a definição da benignidade ou malignidade de neoplasias mamárias das cadelas. Entretanto foi possível inferir através do exame termográfico, realizado no estudo, que a técnica pode auxiliar significantemente no diagnóstico precoce de protocolo terapêutico ao câncer de mama.

\section{Ortopedia Veterinária}

Em pesquisa realizada por INFERNUSO et al. (2010) foi mensurado e comparado padrões térmicos de temperatura na região do joelho de cães clinicamente normais e cães com ruptura do ligamento cruzado cranial (LCCr), antes e após a tosa dos pelos. Embora houvesse aumento significativo na temperatura da pele após a tosa em ambos os grupos, não houve diferença significativa de temperatura entre os joelhos normais e com ruptura do LCCr quando examinados. Desta forma, os autores concluíram que a termografia foi bem-sucedida na detecção da diferenciação que ocorre naturalmente na ruptura do LCCr em cães, não sendo necessário a tosa para o sucesso da avaliação termográfica do joelho canino.

Em outro estudo na área de ortopedia veterinária a termografia mostrouse eficiente para detectar claudicação dos membros pélvicos em cães por meio da observação da impressão deixada ao solo. O objetivo deste estudo foi estabelecer o padrão da impressão termográfica da pegada de cães com claudicação comparada a cães saudáveis. Verificou-se também se há diferenças na temperatura da pegada de membro claudicante versus membro normal de cão normal e membro que suporta o peso de cão claudicante versus membro normal de cão normal. As análises de padrões térmicos das imagens foram bem sucedidos na diferenciação da imagem termográfica da pegada dos cães normais em comparação com cães com claudicação (GARCIA \& SCHOSSLER, 2014). 


\section{Oftalmologia Veterinária}

A termografia infravermelha foi também utilizada na oftalmologia veterinária para mensuração das diferenças de temperaturas da superfície da córnea entre regiões límbicas nasal e temporal e córnea central de cães normais e cães com ceratoconjuntivite seca. Neste mesmo estudo, o exame ainda foi utilizado para determinar os valores de temperatura de olhos caninos normais e em pacientes com baixos valores no teste de lágrima de Schirmer. A temperatura da córnea foi significativamente menor nos olhos com baixos valores no teste de lágrima de Schirmer em relação aos olhos do grupo controle. Desta forma, os autores concluíram que a termografia é um método que pode ser usada para diferenciar olhos com valores normais daqueles com valores anormais no teste de Schirmer (BIONDI et al., 2013).

\section{Anestesiologia Veterinária \\ De acordo com VAINIONPÄÄ et al., (2013), em pesquisa na área de} anestesiologia veterinária, observaram as mudanças de temperatura periférica em cães durante diferentes protocolos de sedação e relacionaram os resultados à mudanças na temperatura retal. A temperatura superficial foi registrada com uma câmera termográfica, tal como indicador de perda de calor periférica potencial em cães. Os autores concluíram que mudanças de temperatura periférica em cães podem ser detectadas pela termografia, e que qualquer protocolo de sedação pode ter efeito sobre imagens termográficas.

\section{Animais Silvestres}

A utilização da termografia em animais silvestres apresenta-se como uma ferramenta de auxilio, permitindo avaliação sem o estresse da contenção e sem risco ao examinador (FRANKS \& PLOWMAN, 2005). Esse método de diagnóstico é utilizado para estudos de estresse ambiental, termorregulação, comportamento, diagnóstico de doenças, visando o bem - estar animal. As aves silvestres são animais que ficam estressadas com presença do homem em seu ambiente, ocasionando alterações na fisiologia térmica e no comportamento (FIGUEIREDO et al., 2012). Nos animais silvestres, de vida livre e os de zoológico é possível com a utilização do aparelho termográfico realizar um exame de qualidade, e com uma certa distância que garanta segurança ao profissional que o executa, principalmente quando os animais examinados são mais hostis (GARNER et al., 1995). A importância do exame, assim como nas outras espécies, consiste no fato de se obter resultados confiáveis, que podem ser utilizados em vários animais ao mesmo tempo, como em uma área aberta (GARNER et al., 1995; FRANKS \& PLOWMAN, 2005). O exame termográfico na medicina veterinária de animais silvestres além de auxiliar na detecção de enfermidades também é utilizado para determinar a população de animais em determinadas áreas, através do sistema de digitalização para o levantamento das populações de espécies de vida silvestre (FRANKS \& PLOWMAN, 2005).

\section{CONSIDERAÇÕES FINAIS}

São diversas as utilidades e benefícios que a termografia oferece na área da medicina veterinária. Apesar desta tecnologia não ser recente, e ser utilizada amplamente na medicina, na engenharia e na área militar, somente nos últimos anos os médicos veterinários começaram a utilizar este método para auxiliar nos diagnósticos, principalmente na área de pesquisa. No entanto, é importante ressaltar 
que como condição necessária para alcançar um diagnóstico seguro, o exame termográfico seja realizado por profissional com o devido treinamento para este procedimento, para correta mensuração e leitura dos termogramas. Necessita-se também que sejam seguidas as exigências mínimas para realização do exame, atendendo desde instalações adequadas, obedecendo aos valores de temperatura estabelecidos, o preparo do paciente e equipamento de alta sensibilidade.

A princípio, a utilização deste aparelho pode ser considerada irrelevante, por muitos profissionais, visto que se aplicam na rotina de atendimento outros métodos de imagem, que são inquestionáveis. Considerando que mesmo sendo utilizado como um método auxiliar de diagnóstico, o seu uso na rotina, tanto em hospitais veterinários quanto em clínicas, agrega valor as consultas realizadas pelos clínicos. Também seria interessante o uso desse exame pelo fato de ser um método não invasivo, indolor, não necessitando do uso de sedativos e por não emitir radiação ionizante. $\mathrm{O}$ uso é viável, possibilitando detectar precocemente enfermidades, auxiliando desta forma um prognóstico favorável aos pacientes independente da espécie. É preciso explorar mais a termografia como um todo, difundir as vantagens, de modo que os médicos veterinários adquiram maior compreensão do real valor que a termografia tem a oferecer nas diversas especialidades da medicina veterinária e na experimentação científica.

\section{REFERENCIAS}

ARORA, N; MARTINS, D; RUGGERIO, D; TOUSIMIS, E; SWISTEL AJ; OSBORNE $_{1}$ MP; SIMMONS, RM. Effectiveness of a noninvasive digital infrared thermal imaging system in the detection of breast cancer. The American Journal Surgery, v.196, n.4, p.523-526, 2008.

BAILEY, S.R; MARR ${ }_{2}$ CM; ELLIOTT, ${ }_{2}$ J. Current research and theories on the pathogenesis of acute laminitis in the horse.Veterinary Journal, v.167, n.2, p.129142, 2004.

BASILE, R.C; BASILE, M.T.; FERRAZ, G.C. Equine inflammatory process evaluation using quantitative thermografic methodology. Ars Vet., v.26, p.77-81, 2010. Disponível

em: http://onlinelibrary. wiley.com/doi/10.1111/vop.12086/abstract;jsessionid=6A3AD2F0B C7D1BD03AAFFF0144FD9916.f03t02

BIONDI, F; DORNBUSCH, PT; SAMPAIO, M; FERREIRA FM. Infrared ocular thermography in dogs with and without keratoconjuctivitis sicca. Veterinary Ophthalmology, p.1-7, 2013.

BRIOSCHI, M.L; MACEDO J.F; MACEDO, C.R.A.Termografia cutânea: novos conceitos. Revista Vascular Brasileira 2003; 2 (2): 151-160.

BOWERS S, GANDY B, ANDERSON P, RYAN P, WILLARD S Assessment of pregnancy in the late-gestation mare using digital infrared thermography. Theriogenology. 72:372-377, 2009. 
CETINKAYA, M.A.; DEMIRUTKU, A. Thermography in the assessment of equine lameness.Turk J Vet AnimSci 36(1): 43-48, 2012.

CHRISTENSEN, J; MATZEN, L.H.; VAETH, M.; SCHOU, S.; WENZELL, A.Thermography as a quantitative imaging method for assessing postoperative inflammation.Dentomaxillofac Radiol, 2012; 41(6): 499.Doi: $10.1259 / \mathrm{dmfr} / 98447974$.

DE LIMA, V. ; PILES, M; RAFEL, O; LÓPEZ-BÉJAR, M; RAMÓN, J; VELARDE, A; DALMAU, A. Infrared thermography to assess the influence of high environmental temperature on rabbits. Research in Veterinary Science, v.95, n.2, p.802-810, 2013.

EDDY, A.L.; VAN HOOGMOED, L.M.; SNYDER, J.R.The role of thermography in the management of equine lameness.The Vet J. 162: 172-181, 2001. Disponível em: http://www.sciencedirect.com/science/article/pii/S1090023301906185.

EDGAR, J. L.; NICOL, C.J.; PUGH, C.A.; PAUL, E.S. Surface temperature changes in response to handling in domestic chickens. Physiology \& Behavior, Elmsford, v. 119, n. 2, p.195-200, 2013.

FERREIRA, V.M.O.S.; FRANCISCO, N.S.; BELLONI, M.; AGUIRRE, G.M.Z.; CALDARA, F.R.; NÄÄS, I.A.; GARCIA, R.G.; ALMEIDA, I.C.L.; POLYCARPO, G.V. Infrared termography applied to the evaluation of metabolic heat loss of chicks fed ith different energy densities. Brazilian Journal of Poultry Science.Campinas, v.13, n.2, p. 113-118, 2011.

FIGUEIREDO, T;DZYEKANSHI, B; KUNZ, J; SILVEIRA, AB; RAMOS, C.M.G; MICHELOTTO, JÚNIOR PV. A importância do exame termográfico na avaliação do aparato locomotor em equinos atletas. Revista Científica Eletrônica de Medicina Veterinária.2012;9(18). Disponível em: http://faef.revista.inf.br/imagens arquivos/arquivos destaque/eLE4dfglj6RTrB2 2013 -6-25-17-23-40.pdf. ISSN: 1679-7353.

FRANKS, M. F.; PLOWMAN, A. Application of remote thermal imaging and night vision technology to improve endangered wildlife resource management with minimal animal distress and hazard to humans.Journal of Physics, p. 207-212, 2005.

GARCIA, E.F.V.; SCHOSSLER, J.E.W . Claudicação em cães - revisão de literatura. Medvep. Revista Científica de Medicina Veterinária. Pequenos Animais e Animais de Estimação , v. 12, p. 100-107, 2014.

GARNER, D.;PORTER, W.F.; UNDERWOOD, H. B. Use of modern thermography for wildlife population surveys. Environmental Management, vol. 19, n. 2, p. 233-238, 1995.

GRACIANO, D. E., NÄÄS, A.I, GARCIA, G.R, CALDARA, R.F. Identificação de artrite em suíno utilizando imagem termográfica. B. Indústr. Anim., Nova Odessa,v.71, n.1, p. 79-3, 2014. 
HENSON, F.M.D. Equine back pathology: diagnosis and treatment. West Sussex: Blackwell Publishing, 2009.

INFERNUSO T, LOUGHIN, CA, MARINO, DJ, UMBAUGH, SE, SOLT, PS. Thermal Imaging of Normal and Cranial Cruciate Ligament-Deficient Stifles in Dogs. Veterinary Surgery 2010, Vol. 39, pp. 410-417.

$\mathrm{KIM}$, J. H.; PARK, H. M. Unilateral femoral arterial thrombosis in a dog with malignant mammary gland tumor: clinical and thermographic findings, and successful treatment with local intra-arterial administration of streptokinase. The Journal of Veterinary Medical Science, v.74, n.5, p.657-661, 2012.

KOTRBACEK, V.; NAU, H.R.The Changes in skin temperatures of periparturient sows.Acta Veterinaria. Brno, v. 54, p.35-40, 1985.

LAHIRI, B.B.; S. BAGAVATHIAPPAN;T. JAYAKUMAR. Medical application of infrared thermography: A review. Infrared Physics \& Technology, v.55, n.4, p.221235, 2012.

LANGE, R. R.; DORNBUSCH, P. T.; PRADO, A. M. R. B. Estudo da aplicação da termografia na doença periodontal em cães. 2014. Dissertação (Mestrado em Programa de Pós Graduação em Ciências Veterinárias) - Universidade Federal do Paraná.

LOUGHIN, C. A, MARINO, D. J. Evaluation of thermographic imaging of the limbs of healthy dogs.American Journal of Veterinary Research, v.68, n.10, p.1064-1069, 2007

MALDAGUE, XP. Advances in pulsed phase thermography.Infrared Physics \& Technology, Maryland, v. 43, p. 174-181, 2002.

MIKAIL, S. Termografia: diagnóstico através da temperatura. Nosso Clínico, v.13, n.74, p.20-24, 2010.

PEREZ DE DIEGO, A.C.; SANCHEZ-CORDON, P.J.; PEDRERA, M.; MARTINEZLOPEZ, B.; GOMEZ-VILLAMANDOS, J.C.; SANCHEZ-VIZCAINO, J.M. The use of infrared thermography as a non-invasive method for fever detection in sheep infected with blue tongue virus. Vet. J., v.198, p.182-186, 2013.

RAINWATER-LOVETT, K.; PACHECO, J.M.; PACKER, C.; RODRIGUEZ, L.L. Detection of foot-and-mouth disease virus infected cattle using infrared thermography.Vet. J., v.180, n.3, p.317 - 324, 2009.

REIS F. R., BARREIRA A. P. B., CASTRO V., CASTRO J. L. C., SUZANO S. M. C., ROCHA A. A. Indícios sobre a correlação entre diferentes métodos diagnósticos em casos de tumor de mama em cadelas. Revista Eletrônica Novo Enfoque09:14-3, 2010.Disponível: http://www.castelobranco.br/sistema/novoenfoque/files/09/artigos/02.pdf. 
RING, EFJ.The discovery of infrared radiation in 1800.ImagingSci J. 2000; 48 (1): 18.Disponível:

http://www.researchgate.net/publication/261098985 The discovery of infrared radi ation in 1800 .

RING, E.F.J. Quantitative thermal imaging.ClinPhysPhysiolMeas11(A):87-95, 1990. ROBERTO JVB, SOUZA BB. Utilização da termografia de infravermelho na medicina veterinária e na produção animal.J Anim Behav Biometeorol. 2014; 2 (3) 73-84. Disponível:

http://periodicos.ufersa.edu.br/revistas/index.php/jabb/article/download/3763/5478. ISSN: 2318-1265./

ROSS, M.W.; DYSON, S.J. Diagnosis and management of lameness in the horse.Ed Saunders, 236-239, 2003.

SANCHEZ B.M, LESCH, M.; BRAMMER, D.; BOVE, S.E.; THIEL, M.; KILGORE,K.S.L. Use of a portable thermal imaging unit as a rapid, quantitative method of evaluating inflammation and experimental arthritis. J Pharmacol Toxicol Methods 57(3): 169-175,2008.

SCHAEFER A. L., DUBESKI P. L., AALHUS J. L., TONG A. K. W. Role of nutrition in reducing antemortem stress and meat quality aberrations. Journal Animal of Science. 79:91-101, 2001.

SCHAEFER AL, COOK NJ, CHURCH JS, BASARAB J, PERRY B, MILLER C, TONG AKW .The use of infrared thermography as an early indicator of bovine respiratory disease complex in calves. Research in Veterinary Science. 83:376384, 2007.

SIMON, E.L.; GAUGHAN, E.M.; EPP, T.; SPIRE, M. Influence of exercise on thermographically determined surface temperatures of thoracic and pelvic limbs in horses.JAm Vet Med Assoc229(12): 1940-1944, 2006.

STEWART, M.; WEBSTER, J.R.; SCHAEFER, A.L.; COOK, N.J.; SCOTT, S.L. Infrared thermography as a non-invasive tool to study animal welfare. Animal Welfare, South Mimms, v.14, p.319-325, 2005.

TUNLEY, B.V.; HENSON, F.M.D. Reliability and repetability of thermographic examination and the normal thermographic image of the thoracolumbar region in the horse.Equine Vet J36(4): 306-312, 2004. Disponível em: http://onlinelibrary.wiley.com/doi/10.2746/0425164044890652/pdf.

TURNER, T.A.; PANSCH, J.; WILSON, J.H. Thermographic assessment of racing thoroughbreds.AAEP Proceedings 47: 344-346, 2001.

TURNER, T. A.; EDDY, L. Diagnóstico pela termografia. Revista Veterinária, no 4, p. 17-95, 2001. 
VAINIONPAA, M.; SALLA, K.; RESTITUTTI, F.; RAEKALLIO, M.; JUNNILA, M.; VAINIO, O. Thermographic imaging of superficial temperature in dogs sedated with medetomidine and butorphanol with and without MK-467.Vet. Anaesth.Analg., v.40, p.142-148, 2013.

VIANNA DML, CARRIVE P. Changes in cutaneous and body temperature during and after conditioned fear to context in the rat. Eur. J. Neurosci. 2005; 21 (9): 2505-12. Disponível em: http://unsworks.unsw.edu.au/fapi/datastream/unsworks:3471/SOURCE1.

VICARI NOGUEIRA, CHF.Termografia por Infravermelho em Cirurgia Plástica Novos Horizontes. PAJMT, v. 1,n.2, 2014. 